\title{
AVALIAÇÃO DA INCIDÊNCIA DE OBESIDADE EM PACIENTES COM DIAGNÓSTICO DE ASMA ATENDIDOS NO AMBULATÓRIO DE PNEUMOPEDIATRIA DE UM HOSPITAL DE ATENÇÃO TERCIÁRIA
}

\author{
Melina Marques Gomes, Osvaldo Saraiva Marques Júnior, Vinícius Marques Gomes \\ Universidade do Oeste Paulista - UNOESTE, Curso de Medicina, Presidente Prudente, SP. \\ E-mail: melinamgomes@hotmail.com
}

\section{RESUMO}

A asma é uma doença inflamatória crônica que gera uma hiper-responsividade das vias aéreas acarretando crises frequentes de sibilância, dispneia, opressão torácica e tosse. Este trabalho teve como objetivo caracterizar o perfil dos pacientes portadores de asma, atendidos em um Hospital de Atenção Terciária para que possam ser implementadas orientações nutricionais a esses pacientes. Trata-se de um estudo transversal, onde foi analisado o índice de massa corpórea em pacientes asmáticos maiores de 6 anos e menores de 18 anos, sendo os dados obtidos por medidas antropométricas e do preenchimento de formulário aplicado durante ambulatório de Pneumopediatria. Observou-se uma maior prevalência de asma na população pediátrica masculina, concordando com a literatura. A taxa de obesidade concomitante com asma foi de 24,67\%, maior do que na literatura. Em parte, tal fato reforça a tendência de associação entre asma e obesidade observada em diversos estudos. O uso de corticoides inalatórios concorda com as preconizações e consenso terapêutico de eficácia no tratamento de crises asmáticas.

Palavras-chave: Asma, Pediatria, Obesidade, Índice de massa corpórea.

\section{EVALUATION OF THE INCIDENCE OF OBESITY IN PATIENTS WITH ASTHMA DIAGNOSIS ATTENDED IN THE PNEUMOPEDIATRY AMBULATORY OF A HOSPITAL OF TERTIARY ATTENTION}

\begin{abstract}
Asthma is a chronic inflammatory disease that causes hyperresponsiveness of the airways leading to frequent bouts of wheezing, dyspnea, chest tightness and cough. The objective of this study was to characterize the profile of patients with asthma treated at a Tertiary Care Hospital so that nutritional guidelines can be implemented for these patients. This is a cross-sectional study, in which the body mass index was analyzed in asthmatic patients over 6 years of age and under 18 years of age. The data were obtained by anthropometric measurements and the form filled out during a Pneumopediatrics outpatient clinic. A higher prevalence of asthma was observed in the male pediatric population, in agreement with the literature. The rate of obesity concomitant with asthma was $24.67 \%$, higher than in the literature. In part, this fact reinforces the tendency of association between asthma and obesity observed in several studies. The use of inhaled corticosteroids agrees with the recommendations and therapeutic consensus of efficacy in the treatment of asthmatic crises.
\end{abstract}

Keywords: Asthma, Pediatrics, Obesity, Body mass index. 


\section{INTRODUÇÃO}

A asma é uma doença inflamatória crônica que atinge o trato respiratório. A inflamação ocasionada pela doença gera uma hiper-responsividade das vias aéreas acarretando crises frequentes de sibilância, dispneia, opressão torácica e tosse principalmente à noite e ao despertar. As exacerbações são passageiras e ocorrem devido à obstrução das vias aéreas, porém, o processo inflamatório é persistente mesmo fora do período de crises. Os episódios podem ser revertidos espontaneamente ou controlado através do uso de medicamentos ${ }^{1}$.

A asma afeta cerca de 300 milhões de indivíduos em todo o mundo. No Brasil, existem aproximadamente 20 milhões de asmáticos, se for considerada uma prevalência global de $10 \%{ }^{2}$. Os pacientes com asma grave representam apenas 5\%-10\% dos casos, porém apresentam maior morbimortalidade relativa, e são responsáveis por um consumo exageradamente alto dos recursos de saúde em relação aos grupos de menor gravidade. Portadores de asma grave não controlada procuram 15 vezes mais as unidades de emergência médica e são hospitalizados 20 vezes mais que os asmáticos moderados ${ }^{3}$.

A etiopatogenia da asma envolve uma série de reações inflamatórias mediadas por inúmeras células imunológicas. Esse mecanismo leva a contração da musculatura lisa brônquica, ao edema da mucosa e a hipersecreção, que juntos acarretam o estreitamento dos brônquios, sendo este intermitente e reversível. Porém, a hiper-responsividade brônquica e resposta exagerada aos estímulos podem ser irreversíveis e levar ao remodelamento em longo prazo ${ }^{1}$.

O diagnóstico da asma deve ser baseado na anamnese e exame físico e sempre que possível nas provas de função pulmonar ${ }^{4}$. $\mathrm{Na}$ anamnese são indicativos de asma: um ou mais dos sintomas dispneia, tosse crônica, sibilância, aperto no peito ou desconforto torácico, particularmente à noite ou ao despertar; sintomas episódicos; melhora espontânea ou pelo uso de medicações para asma; três ou mais episódios de sibilância no último ano; variabilidade sazonal dos sintomas e história familiar positiva para asma; e diagnósticos alternativos excluídos 5 .

O exame físico frequentemente é normal na intercrises, mas o achado mais característico e frequente é a presença de sibilos na ausculta, especialmente na expiração forçada ${ }^{2}$.

As provas de função pulmonar informam sobre a intensidade da limitação ao fluxo aéreo, sua reversibilidade e variabilidade. A espirometria é útil para diagnóstico, avaliação da gravidade, monitorização e avaliação da resposta ao tratamento. Ela deve ser utilizada nas crianças a partir dos cinco anos. O volume expiratório forçado no primeiro segundo (VEF1) pós-broncodilatador é o melhor parâmetro espirométrico para avaliar mudanças em longo prazo na função pulmonar, sendo um indicador de progressão da doença. A medida do pico de fluxo expiratório (PFE) serve para avaliar a variabilidade da obstrução; auxilia a monitorização clínica e a detecção precoce de crises $^{3}$. Segundo a Sociedade Brasileira de Pediatria os achados funcionais pulmonares compatíveis com a asma são um aumento de VEF1 maior que $12 \%$ e maior que $200 \mathrm{~mL}$ depois da administração de broncodilatador. Nos pacientes menores de 2 anos o diagnóstico é clínico ${ }^{1}$.

A asma pode ser classificada da seguinte forma segundo a Global Initiative of Asthma, bem controlada, parcialmente controlada e não controlada de acordo com uma avaliação feita nas últimas 4 semanas. O paciente deve responder a quatro perguntas: - Possui sintomas diurnos mais do que 2 vezes por semana; - Possui despertares noturnos; - Necessitou usar medicação de alívio mais do que duas vezes por semana; - Possui alguma limitação de atividade em decorrência da asma. Se a resposta for não para todas elas à asma é bem controlada, se o paciente responder sim a uma ou duas dessas perguntas a asma será parcialmente controlada e se responder sim a três ou mais a asma será não controlada².

Existem duas formas de tratamento medicamentoso da asma, o tratamento para a manutenção e o tratamento para as crises. O tratamento de manutenção é baseado no uso de corticoides inalatórios. Há numerosas evidências de que eles são efetivos no controle dos sintomas da asma e na redução da intensidade da inflamação das vias aéreas, e de que esses 
efeitos perduram durante o tempo de corticoterapia ${ }^{6}$. Além de controlar os sintomas da asma e de melhorar a função respiratória, os corticoides previnem as exacerbações e reduzem a mortalidade da asma ${ }^{7}$. Seus efeitos independem da idade do doente e da gravidade da doença ${ }^{8}$.

Segundo a Global Initiative for Asthma baixas doses regulares de corticoide inalatório são recomendadas para todos os pacientes com diagnóstico de asma e qualquer um dos seguintes: sintomas de asma mais do que duas vezes por mês, acordar com sintomas de asma mais do que uma vez ao mês e qualquer sintoma de asma juntamente com qualquer fator de risco para exacerbações ${ }^{2}$.

Recentes estudos passaram a associar a asma com obesidade, principalmente nas nações desenvolvidas. Segundo esses estudos ocorre uma maior prevalência de asma e obesidade principalmente em meninas e adolescentes do sexo feminino?

Por ser uma doença muitas vezes limitante e crônica, a asma, junto com o sedentarismo contribui imensamente para a chamada "epidemia de obesidade infantil" que vem ocorrendo no mundo todo. Além disso, a obesidade parece estar associada ao pior controle da doença, com frequente exacerbação dos sintomas e redução da tolerância ao exercício físico. Da mesma forma, o aumento de peso também pode causar uma desvantagem na mecânica pulmonar, levando à diminuição da capacidade funcional, volume pulmonar e do diâmetro das vias aéreas ${ }^{10}$.

As repercussões da asma e da obesidade atingem não somente o paciente, mas todo o universo familiar ${ }^{11}$, podendo trazer problemas complexos e implicações em longo prazo, interferindo assim na qualidade de vida desses indivíduos ${ }^{10}$. Os efeitos causados pela asma podem prejudicar o bem-estar físico e emocional do paciente, podendo inclusive acarretar consequências em seu rendimento escolar ${ }^{12}$.

A obtenção desses dados é de suma importância para determinar a dimensão do impacto da enfermidade na saúde da criança como um todo ${ }^{10}$.

Desta forma, a relevância deste estudo encontra-se na importância de traçar um perfil dos pacientes asmáticos atendidos no ambulatório de Pneumopediatria de um Hospital de Atenção Terciária para que de acordo com os resultados possam ser implementadas orientações nutricionais a esses pacientes.

\section{METODOLOGIA}

Trata-se de um estudo transversal, onde serão analisados os Índices de Massa Corpórea de pacientes com diagnóstico de asma maiores de 6 anos e menores de 18 anos, atendidos no ambulatório de pneumopediatria de um hospital de Presidente Prudente/SP, no período de fevereiro a maio de 2018. Foram excluídos os menores de 6 anos devido à dificuldade de diagnóstico da asma em tais pacientes.

Após explicitação detalhada do propósito do estudo, bem como de suas limitações, os responsáveis assinaram o Termo de Consentimento Livre Esclarecido (TCLE) e o Termo de Assentimento e foram colhidas as medidas antropométricas e preenchimento de formulário.

No estudo foram observados os critérios preconizados na Resolução 466/12 do Conselho Nacional de Saúde (CNS), que regulamenta as normas éticas para pesquisa com seres humanos no Brasil, garantindo o sigilo das informações coletadas dos pacientes e seus dados pessoais. $O$ projeto foi cadastrado na Plataforma Brasil e submetido ao Comitê de Ética (CAAE 87939618.0.0000.5515), para sua análise e aprovação. O protocolo de pesquisa envolveu os seguintes itens: sexo, idade, peso, altura, índice de massa corpórea, uso de corticoide inalatório e uso de corticoide oral no último ano. As medidas antropométricas foram tomadas, de acordo com a Organização Mundial de Saúde ${ }^{13,14}$.

Utilizou-se uma balança com capacidade para até $100 \mathrm{~kg}$ para aferição do peso e verificouse a estatura por meio de um antropômetro com escala de precisão de $0,1 \mathrm{~cm}$. Posicionou-se o paciente sobre a base do antropômetro, estando descalço, ereto, com o peso do corpo distribuído 
igualmente sobre ambos os pés, colocando as superfícies posteriores dos calcanhares, as nádegas e a região occipital em contato com a escala de medida, e com a cabeça orientada de modo que a linha de visão ficasse perpendicular ao corpo. A referência para mensuração foi o ponto mais alto da cabeça com pressão suficiente para comprimir o cabelo ${ }^{14}$.

A classificação do estado nutricional foi realizada de acordo com os novos critérios adotados pela literatura e utilizando-se os indicadores peso-para-idade $(\mathrm{P} / \mathrm{I})$, estatura-para-idade $(E / I)$, peso-para-estatura $(P / E)$, e índice de massa corporal para a idade $(I M C / I)^{13,14}$.

\section{RESULTADOS}

Foram incluídos no estudo 60 pacientes, dos quais 36 eram do sexo masculino (60\%) e 24 do feminino (40\%) (FIGURA 1). As idades variaram entre 6 e 17 anos, com a média de 10,6 anos ( $[D P]= \pm 3,02$ ) (FIGURA 2); o cálculo do coeficiente de assimetria resultou em 0,31 (para beta) e 0,32 (para gama) e um teste de normalidade de acordo com Shapiro-Wilk de 0,021 (W=0,95).

O peso dos pacientes variou entre 17 e $89 \mathrm{~kg}$, com a média de 44,8 kg (Desvio-Padrão [DP] $= \pm 16,26$ ) (FIGURA 3); o cálculo do coeficiente de assimetria resultou em 0,45 (para beta) e 0,46 (para gama) e um teste de normalidade de acordo com Shapiro-Wilk de 0,10 ( $W=0,96)$. Foram identificadas 13 crianças obesas (21,67\%).

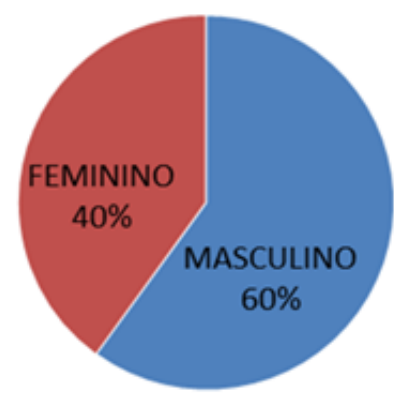

Figura 1. Distribuição dos pacientes de acordo com o sexo. Fonte: Dados da Pesquisa.

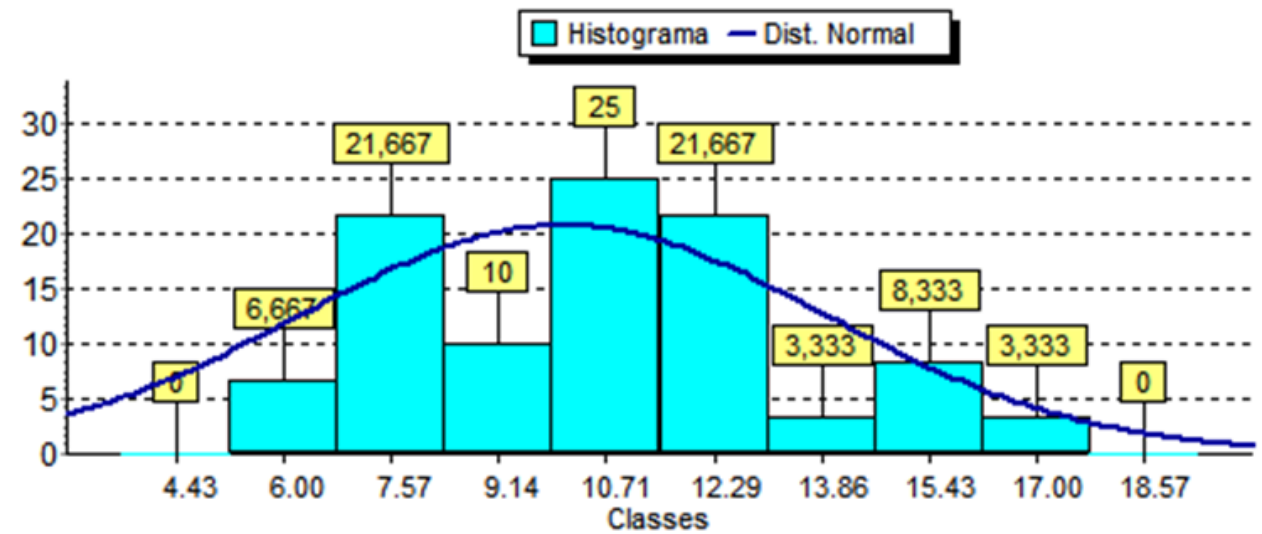

Figura 2. Histograma de Frequência das Idades dos Pacientes.

Fonte: Dados da Pesquisa. 


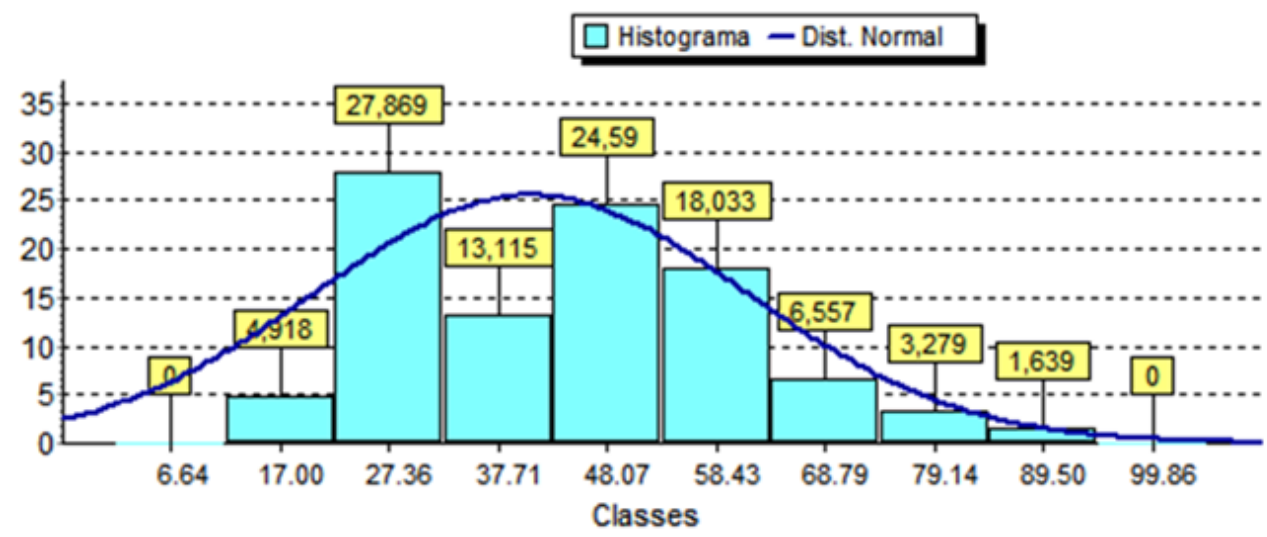

Figura 3. Histograma de Frequência do Peso dos Pacientes.

Fonte: Dados da Pesquisa.

A altura dos pacientes variou entre 1,06 e 1,80 metros, com a média de 1,46 metros ([DP] $=$ $\pm 0,17$ ) (FIGURA 4); o cálculo do coeficiente de assimetria resultou em $-0,30$ (para beta) e $-0,31$ (para gama) e um teste de normalidade de acordo com Shapiro-Wilk de 0,23 (W=0,97).

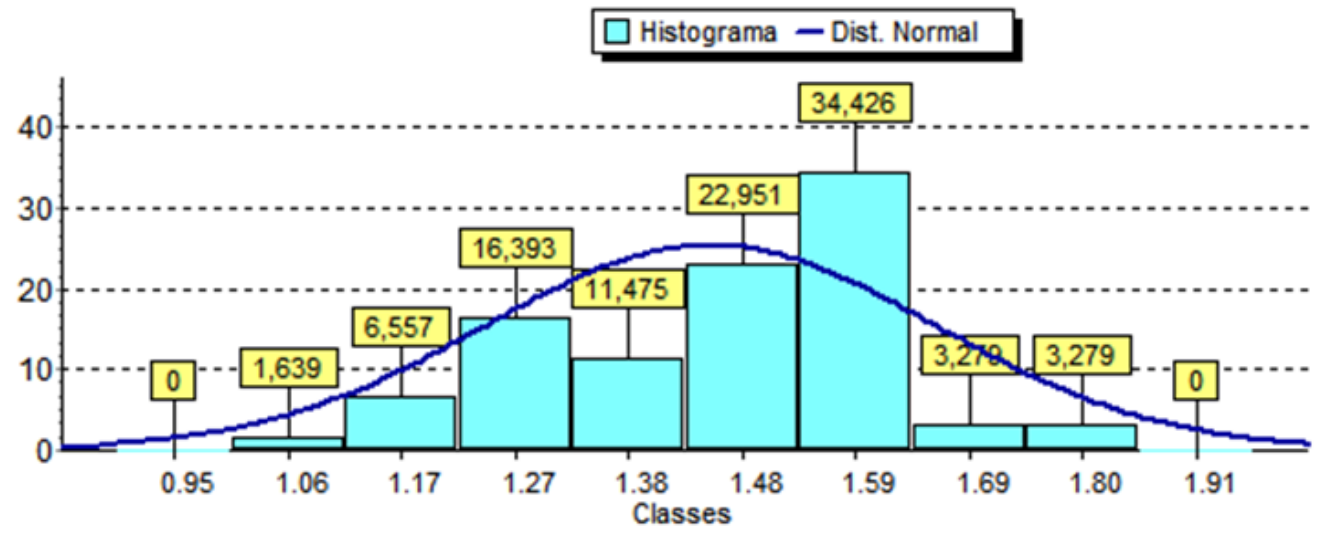

Figura 4. Histograma de Frequência das Alturas dos Pacientes.

Fonte: Dados da Pesquisa.

O Índice de Massa Corporal (IMC) variou entre 15,1 e $35 \mathrm{~kg} / \mathrm{m}^{2}$, com a média de $20,6 \mathrm{~kg} / \mathrm{m}^{2}$ (Desvio-Padrão $[D P]= \pm 4,6$ ) (FIGURA 5); o cálculo do coeficiente de assimetria resultou em 1,08 (para beta) e 1,12 (para gama) e um teste de normalidade de acordo com Shapiro-Wilk de 0,003 $(\mathrm{W}=0,91)$. O percentil do IMC variou entre $1 \%$ e $99 \%$, com a média de $71 \%$.

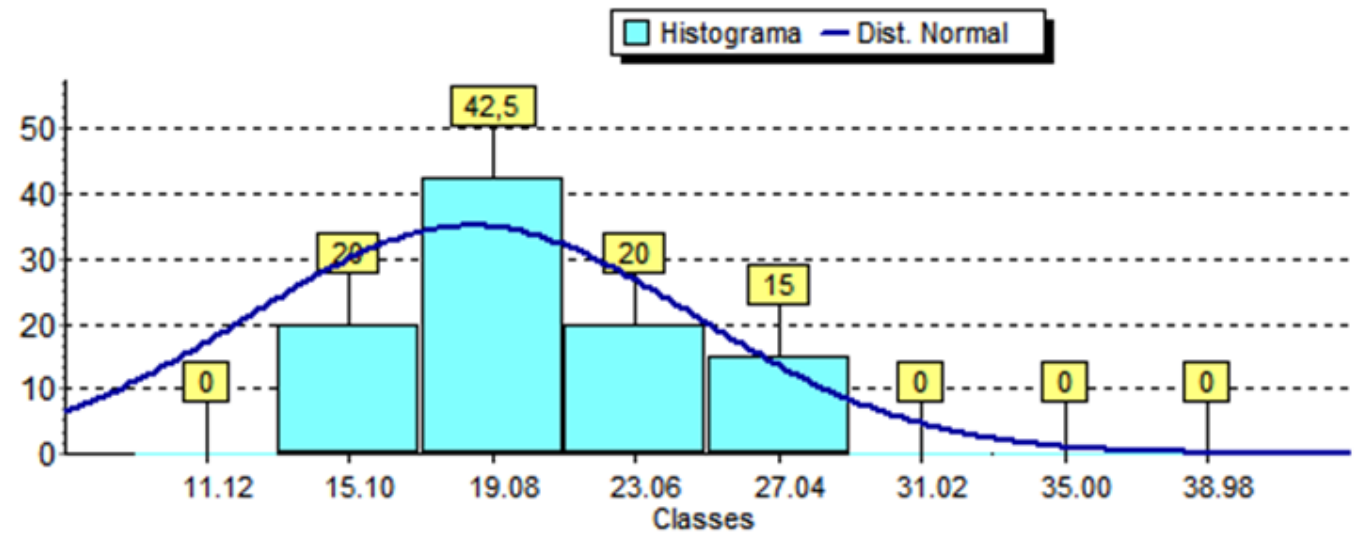

Figura 5. Histograma de Frequência do IMC dos Pacientes.

Fonte: Dados da Pesquisa. 
Entre os pacientes, 38 faziam uso de corticoides inalatórios (63,33\%) e 22 não (36,67\%). Já entres os usuários de corticoides orais no último ano, 6 fizeram uso (10\%), dentre os quais um era de uso contínuo, e 54 não fizeram uso (90\%) (TABELA 1).

Tabela 1. Uso de corticoides pelos pacientes.

\begin{tabular}{ccccc}
\hline \multicolumn{5}{c}{ Uso de Corticoides por Pacientes } \\
\hline & Corticoides Inalatórios & \multicolumn{2}{c}{ Corticoides Orais } \\
SIM & $\mathrm{N}$ & $\%$ & $\mathrm{~N}$ & $\%$ \\
NÃO & 38 & 63,33 & 6 & 10 \\
TOTAL & 22 & 36,67 & 54 & 90 \\
\hline
\end{tabular}

Fonte: Dados da Pesquisa.

\section{DISCUSSÃO}

A asma e obesidade tem tido um aumento em sua prevalência a nível mundial, levando a comunidade científica a analisar esta relação de forma mais íntima ${ }^{15}$.

O presente estudo revelou uma maior prevalência no sexo masculino (60\%), tal dado corrobora com outro estudo ${ }^{16}$, onde a prevalência da incidência de asma em crianças do sexo masculino era de 70,6\%. Entretanto na comparação com tal estudo a média de idade encontrada foi maior (10,6 anos). Tal fato explica-se pelas diferenças geográficas e pela amplitude das idades incluídas nos estudos de forma individual. Tal prevalência maior no sexo masculino também é reforçada em metanálise realizada em outro trabalho ${ }^{17}$, porém com porcentagem menor, visto as diferenças geográficas e populacionais.

No estudo já citado ${ }^{16}$, a incidência de obesidade na população pediátrica estudada foi de $8,2 \%$, inferior a taxa encontrada no presente estudo de $21,67 \%$. Tal expressiva incidência sugere e reforça uma relação relevante entre a obesidade e a asma em crianças. Vários estudos tem descrito o "fenótipo obeso-asma", entretanto não é muito claro o seu significado, além de demonstrar que a severidade dos sintomas da asma é maior em pacientes obesos ${ }^{18}$.

Em outro recente estudo do tipo caso-controle, foi descrita uma possível associação entre a obesidade central e a asma em criança e adolescentes, com um Odds-Ratio de 1,52 em relação ao controle utilizado ${ }^{19}$.

Dentre os fatores que relacionam a obesidade à asma ressaltam a maior exposição a alérgenos, a fumaça do tabaco e a alimentação menos saudável, pelo sedentarismo e maior tempo dentro de suas residências ${ }^{20}$. Altos índices concomitantes de obesidade e asma podem relacionarse com o fato da primeira produzir manifestações que mimetizam a segunda, como dispneia, aumento do esforço respiratório, apneia do sono, hipoventilação e refluxo gastroesofágico ${ }^{21}$.

Diante da alta incidência da utilização de corticosteroides inalatória (63,33\%), afirma que a corticoterapia por tal via está bem estabelecida na terapêutica, pois associa-se com uma diminuição dos sintomas em pouco tempo, além de ter efeito sobre a inflamação e melhorar a função pulmonar. Entretanto, um trabalho de metanálise percebeu-se uma tendência a uma ligeira diminuição no crescimento e altura, mesmo que incerta, devido a carência nos estudos observacionais sobre a temática ${ }^{22}$.

Outro risco inerente à terapia com corticosteroides inalatórios é o incremento da colonização orofaríngea por Streptococcus pneumonia ${ }^{23}$.

Em comparação com outro estudo ${ }^{24}$, o uso de corticosteroides via inalatória foi maior, pois neste somente $45,1 \%$ da população estudada fez uso do mesmo. Já em relação ao corticosteroide oral, o presente estudo teve uma taxa menor (10\%), quando comparado ao estudo citado (38\%). 


\section{CONCLUSÃO}

Diante dos dados obtidos, observou-se uma maior prevalência de asma na população pediátrica masculina, concordando com a literatura. Entretanto, a média etário foi maior em decorrência dos critérios de inclusão. A taxa de obesidade concomitante com asma na população estudada foi alta (24,67\%) e maior do que o encontrado na literatura. Em parte, tal fato reforça a tendência de associação entre asma e obesidade observada em diversos estudos, porém não julga nula a hipótese da associação relacionar-se a sintomas miméticos de asmas produzidos por disfunções respiratórias nos obesos. O uso de corticoides inalatórios concorda com as preconizações e consenso terapêutico de eficácia no tratamento de crises asmáticas, entretanto o uso de corticoides orais mostrou-se menor do que na literatura geral. Recomenda-se a realização de estudos experimentais, que avaliem os mecanismos genéticos e fisiopatológicos que indiquem a relação real entre obesidade e asma em população pediátrica, a fim de permitir estratégias de política pública e terapêuticas eficazes em relação a tais afeç̧ões.

\section{REFERÊNCIAS}

1- Lopez FA. Tratado de Pediatria. Soc. Bras. de Pediatr. 2014; 3.

2 - Pocket Guide for Asthma Management and Prevention. Global Initiative for Asthma, 2017.

3 - Protocolo Clínico e Diretrizes Terapêuticas. ASMA. 2013; 1.317,

4 - Tarantino AB. Doenças pulmonares. Guanabara Koogan. 2008;6.

5 - Jornal Brasileiro De Pneumologia. Diretrizes da Sociedade Brasileira de Pneumologia e Tisiologia para o Manejo da Asma. 2012;38.

6 - Zheng S. The influence of inhaled corticosteroid discontinuation in children with well-controlled asthma. Medicine. 2017;96. https://doi.org/10.1097/MD.0000000000007848

7 - Marchioro J. Nível de controle de asma e sua relação com uso de medicação em asmáticos no Brasil. J. Bras. Pneumol. 2014: 40.

8 - Campos HS. Asma: suas origens, seus mecanismos inflamatórios e o papel do corticosteroide. Rev. Bras. de Pneum. San. 2007;15.

9 - Azizpour Y. Efeito do IMC infantil na asma: uma revisão sistemática e meta-análise de estudos caso-controle. BMC Pediatr. 2018;18.

10 - Mendes AA. Estado nutricional antropométrico e qualidade de vida em escolares com asma. Scientia Medica. 2016:26. https://doi.org/10.15448/1980-6108.2016.4.24492

11 - Trinca MA, Bicudo IMP, Pelicioni MCF. Interferência da asma no cotidiano das crianças. Rev. Bras. Crescimento Desenvolv. Hum. 2011;21.

12 - Gazzotti MR. Nível de controle da asma e seu impacto nas atividades de vida diária em asmáticos no Brasil. Jornal Brasileiro de Pneumologia. 2013;39.

13 - World Health Organization. Child Growth Standards. Lenght/height-for age, weight-for-age, weight-for-lenght, weight-for-height and body mass index-for-age: methods and development. 2006:312p. 
14 - World Health Organization. Expert Committee on Physical Status: the use and interpretation of anthropometry Physical status: report of a Who expert Committee. 1995:453p.

15 - Lang JE. Obesity, nutrition, and asthma in children. Pediatr. Allergy Immunol. Pulmonol. 2012. https://doi.org/10.1089/ped.2011.0137

16 - Renosto LC. Growth velocity and weight gain in prepubertal asthmatic children. Rev. Assoc. Med. Bras. 2017;63. https://doi.org/10.1590/1806-9282.63.03.236

17 - Aiello AM. Prevalence of Obesity in Children and Adolescents in Brazil: A Meta-analysis of Cross-sectional Studies. Curr Pediatr Rev. 2015;11.

18 - Lotvall J. Asthma endotypes: a new approach to classification of disease entities within the asthma syndrome. J. Allergy Clin. Immunol. 2011; 127.

19 - Papoutsakis C. Associations between central obesity and asthma in children and adolescents: a case-control study. J. Asthma. 2015;52. https://doi.org/10.3109/02770903.2014.954291

20 - Chinn S, Rona RJ. Can the increase in body mass index explain the rising trend in asthma in children? Thorax. 2001;56. https://doi.org/10.1136/thorax.56.11.845

21 - Chen Y. Obesity may increase the incidence of asthma in women but not in men; longitudinal observations from the Canadian National Population Health Surveys. American Journal Epidemiol. 2002;155. https://doi.org/10.1093/aje/155.3.191

22 - Loke YK. Impact of Inhaled Corticosteroids on Growth in Children with Asthma: Systematic Review and Meta-Analysis. PLoS One. 2015;10. https://doi.org/10.1371/journal.pone.0133428

23 - Zhang L. Inhaled corticosteroids increase the risk of oropharyngeal colonization by Streptococcus pneumoniae in children with asthma. Respirology. 2013;18.

24 - Alvim CG. Corticoide oral e inalatório para tratamento de sibilância no primeiro ano de vida. J. Pediatr. (Rio J.). 2011; 87. 